\section{(a)}

\section{Revista Mexicana de Ciencias Forestales}

ISSN: 2007-1132

ciencia.forestal2@inifap.gob.mx

Instituto Nacional de Investigaciones

Forestales, Agrícolas y Pecuarias

México

Waizel-Bucay, José; Cruz-Juárez, María de Lourdes

Arnica montana L., planta medicinal europea con relevancia

Revista Mexicana de Ciencias Forestales, vol. 5, núm. 25, septiembre-octubre, 2014, pp.

98-109

Instituto Nacional de Investigaciones Forestales, Agrícolas y Pecuarias

Distrito Federal, México

Disponible en: http://www.redalyc.org/articulo.oa?id=63439013008

Cómo citar el artículo

- Número completo

- Más información del artículo

- Página de la revista en redalyc.org

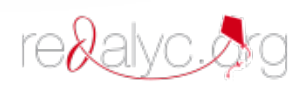

Sistema de Información Científica

Red de Revistas Científicas de América Latina, el Caribe, España y Portugal Proyecto académico sin fines de lucro, desarrollado bajo la iniciativa de acceso abierto 


\title{
Arnica montana L., planta medicinal europea con relevancia Arnica montana L., relevant European medicinal plant
}

José Waizel-Bucay’ y María de Lourdes Cruz-Juárez²

\section{Resumen}

Se exponen diferentes aspectos relativos a la especie Arnica montana (Asteraceae), importante planta medicinal de uso ancestral; a pesar de ser endémica de Europa central y meridional se ha logrado introducir y cultivar con éxito en Estados Unidos de América y Escocia. Respecto a la conservación de la especie cabe mencionar que está bajo estricta protección y se incluye en diversos libros y listas rojas de algunos países europeos, así como en la Lista Roja de Especies Amenazadas de la IUCN. Se presenta un panorama general de A. montana desde el punto de vista científico (descripción botánica, distribución geográfica y sinonimia científica y nombres comunes en distintos idiomas). Debido a que se utiliza para aliviar muchos padecimientos y a que tiene una diversidad de aplicaciones, se mencionan sus usos medicinales, cosméticos, ornamentales y en perfumería. Su historia, las regiones que se dedican a su comercialización y su importancia económica justifican el interés de realizar estudios que aseguren las condiciones adecuadas para su introducción al cultivo en México; como contribución a este tema se presentan las formas de propagación y los requerimientos para su plantación. También se proporcionan datos sobre su fitoquímica, toxicología, contraindicaciones y efectos secundarios. Es de resaltar que A. montana produce numerosos metabolitos secundarios con actividad biológica, pertenecientes a los grupos de los aceites esenciales, ácidos fenólicos, cumarinas, flavonas, flavonoides, glucósidos, mucílagos, fitosteroles, lactonas sesquiterpénicas, taninos y triterpenos, entre otros.

Palabras clave: Arnica montana L., Asteraceae, etnobotánica, farmacognosia, metabolitos secundarios, plantas medicinales.

\begin{abstract}
Different aspects of Arnica montana L. (Asteraceae) are presented in this work. It is an important perennial medicinal herb used since ancient times; it is endemic to central and southern Europe and has been successfully introduced and cultivated in several countries, such as the United States of America, Scotland, and others. It is under strict protection and is included in the IUCN Red List of Threatened Species, in the Red Data Books, and Red Data Lists of many European countries. Method: we applied the technique of direct, active reading of specialized documentary sources such as books, magazines and electronic databases. Results: A descriptive overview of the species: its botanical description, geographic distribution, scientific synonyms and common names in different languages; its history, its phytochemistry, its various uses: medicinal, cosmetic and ornamental; its toxicological aspects and its counter-indications or side effects. We also provide information regarding its economic importance, as well as data about its form of propagation. Furthermore, we include the requirements for its cultivation and the main countries that trade it. The plant produces numerous secondary metabolites with biological activity, including essential oils, phenolic acids, coumarins, flavones, flavonoids, glycosides, mucilages, phytosterols, sesquiterpene lactones, tannins and triterpenoids.
\end{abstract}

Key words: Arnica montana L., Asteraceae, ethnobotany, pharmacognosy, secondary metabolites, medicinal plants.

\footnotetext{
Fecha de recepción/ date of receipt: 11 de febrero de 2014; Fecha de aceptación/date of acceptance: 10 de julio de 2014
}

' Escuela Nacional de Medicina y Homeopatía. Instituto Politécnico Nacional. Correo-e: josewaizel@hotmail.com 
Arnica montana $L$. es una planta con importancia económica para numerosos países debido a su uso como remedio medicinal (en fitoterapia humana y veterinaria y en homeopatía), como materia prima para la elaboración de productos cosméticos y en la fabricación de algunos licores europeos (Benedictine ${ }^{\oplus}$, Calvados $^{\circledast}$, Chartreuse ${ }^{\circledast}$, Coriandro $^{\circledast}$, etcétera.). En Estados Unidos de América y en Europa también se ha documentado su utilización en jardinería, acompañada por 12 especies más del género Arnica (Díaz, 1976; Martínez, 1979; Waizel, 1995; Conabio, 2011)

El uso medicinal del árnica proviene de sus características como astringente y estimulante del corazón y del sistema inmunológico; además, alivia el dolor, actúa como agente antiinflamatorio y es útil en las infecciones bacterianas y fúngicas; es por ello que durante la Segunda Guerra Mundial fue utilizada, ampliamente, para tratar enfermedades de los soldados. Sus hojas se fumaban en Francia, Suecia y Rusia como sustituto del tabaco y sus extractos alcohólicos eran inhalados por personas que deseaban dejar de consumirlo; esto pudo haber originado la costumbre de algunos pastores españoles por aspirarla, sin embargo, se desconoce si había alguna asociación con motivos curativos (Díaz, 1976; Martínez, 1979; Waizel, 1995; Conabio, 2011). Dada su amplia utilización como planta medicinal en ocasiones es adulterada con inflorescencias de otras plantas de la misma familia (Asteraceae, Compositae), sobre todo con Calendula officinalis L., Inula britannica L., Tragopon pratensis L. y Scorzonera humilis L. (Grieve, 1982; Waizel, 1995).

El nombre "árnica" está muy extendido e incluye otros taxones; por ejemplo, Obón et al., 2012 documentaron 34 especies en la herbolaria de la Península lbérica pertenecientes a siete familias comercializadas bajo esa denominación. En México, son varios los taxa que se venden con fines medicinales y se conocen como árnica, árnica de la costa, o árnica del país, estas son Gaillardia spp.; Helenium mexicanum Kunth, Heterotheca inuloides Cass., Heterotheca leptoglossa DC., Neurolaena lobata (L.) Cass., Tithonia diversifolia (Hemsl.) A. Gray, Trixis angustifolia D.C., Trixis radialis (L.) Kuntze, Verbesina crocata (Cav.) Less, Verbesina pinnatifida Sw., Zexmenia pringlei Greenm. y Mentzelia conzattii Greenm. (Díaz, 1976; Martínez, 1979; Waizel, 1995; Conabio, 2011 ).

Otros representantes del género Arnica, nativos de las montañas Rocallosas en el oeste de los Estados Unidos de América, han sido aceptados como curativos y se inscribieron en los formularios medicinales VII y VIII de ese país (Díaz, 1976; Martínez, 1979; Waizel, 1995; Conabio, 2011): A. fulgens Pursh, A. sororia Greene y A. cordifolia Hook. También se ha documentado A. chamissonis Less. (de las estepas de Norteamérica) como materia prima equivalente a A. montana (Nowak, 2002).

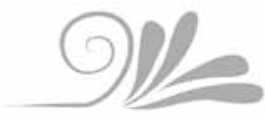

Arnica montana $L$. is an economically important plant for many countries due to its use as a medicinal remedy (in human and veterinarian phytotherapy, as well as in homeopathy), as a raw material for cosmetics and for certain European liquors (Benedictine ${ }^{T M}$, Calvados $^{T M}$, Chartreuse $^{T M}$, Coriandro $^{T M}$, etc.). In the United States of America and also in Europe, its use in gardening has been documented, along with 12 other species of the genus Arnica (Díaz, 1976; Martínez, 1979; Waizel, 1995; Conabio, 2011).

The medicinal use of arnica stems from its characteristics as an astringent and a stimulant for the heart and the immune system; furthermore, it relieves pain, acts as an anti-inflammatory agent and is useful against bacterial and fungal infections; for this reason, it was widely used during World War II to treat the soldiers. Its leaves were smoked in France, Sweden and Russia as a substitute for tobacco, and its alcoholic extracts were inhaled by those who wished to stop smoking. This may have originated the habit, among Spanish shepherds, of inhaling it; however, it is not known whether this is associated in any way to healing purposes (Díaz, 1976; Martínez, 1979; Waizel, 1995; Conabio, 2011). Given its widespread use as a medicinal plant, it is occasionally adulterated with inflorescences of other species of the same family (Asteraceae, Compositae), particularly with Calendula officinalis L., Inula Britannica L., Tragopon pratensis L. and Scorzonera humilis L. (Grieve, 1982; Waizel, 1995).

The name "arnica" is very widespread and includes other taxa; e.g. Obón et al. (2012) documented 34 species among medicinal herbs in the Iberian Peninsula, belonging to seven families that have been commercialized under that name. In Mexico, various taxa are sold for medicinal purposes and are all known as arnica, coastal arnica or local arnica; these are Gaillardia spp., Helenium mexicanum Kunth, Heterotheca inuloides Cass., Heterotheca leptoglossa DC., Neurolaena lobata (L.) Cass., Tithonia diversifolia (Hemsl.) A. Gray, Trixis angustifolia D.C., Trixis radialis (L.) Kuntze, Verbesina crocata (Cav.) Less, Verbesina pinnatifida Sw., Zexmenia pringlei Greenm., and Mentzelia conzattii Greenm. (Díaz, 1976; Martínez, 1979; Waizel, 1995; Conabio, 2011 ).

Other representatives of the Arnica genus which are native of the Rocky Mountains of western USA have been accepted as medicinal and have been registered in the medical formularies VII and VIII of that country (Díaz, 1976; Martínez, 1979; Waizel, 1995; Conabio, 2011): A. fulgens Pursh, A. sororia Greene and A. cordifolia Hook. A. chamissonis Less. (which grows in the steppes of North America) has also been documented as a raw material equivalent to A. montana (Nowak, 2002).

\section{Medicinal and cosmetological uses}

The heads of the inflorescences, the complete plant or the rhizome are used in the industry of cosmetics. The harvest of the first takes place when the capitula begin to open, in late spring 


\section{Usos medicinales y cosmetológicos}

Las cabezuelas de las inflorescencias, el vegetal completo o el rizoma son utilizados en la industria cosmética. La cosecha de las primeras se realiza al comienzo de la apertura de los capítulos, a finales de la primavera o principios del verano; la de los rizomas a principios del otoño, cuando el ejemplar entra en latencia, se deben desarraigar, limpiar y trozar (Herbotecnia, s/f.).

Los productos para uso interno se preparan en forma de elixir - en presentación de glóbulos, gránulos, comprimidos, pomadas, soluciones inyectables, gotas y ampolletas bebiles como remedio homeopático; para uso externo se elaboran cataplasmas, colutorios, cremas tópicas, elixires, sprays, gargarismos, geles, linimentos, parches, pomadas, solución capilares, sticks o ungüentos. La planta seca se emplea en la fitoterapia tradicional (con las debidas precauciones por la toxicidad que presenta), como infusión, cocimiento o decocción, tinturas, lociones, entre otras (CEDICRAT, 2009).

\section{Descripción botánica}

Arnica montana es una hierba perenne, de 20 a $60 \mathrm{~cm}$ de altura, con hojas inferiores (basales) enteras situadas a ras del suelo, de color verde brillante, ligeramente vellosas en la superficie superior, agrupadas en racimos (rosetas planas), ovado-ciliadas y con puntas redondeadas. Las hojas superiores son más pequeñas, opuestas, de forma lanceolada, y están adheridas directamente al tallo subterráneo. Desde el centro de la roseta, emerge un tallo redondeado y piloso que concluye en uno a tres tallos florales que terminan en una inflorescencia hermafrodita con forma de cabezuela, de siete a ocho cm de diámetro y, por excepción, de uno a tres pares de cabezuelas menores, en sendas ramitas laterales, cada una de las cuales está constituida por una serie de flores periféricas, liguladas, compuestas por un cáliz, representado por cerdas erizadas y de puntas finas, con una corola zigomorfa, formada por una lámina de tres dientes y un ovario terminado en un apéndice bifurcado en la punta. Hacia el centro de la cabezuela se encuentran las flores centrales, con la corola tubulosa o quinquedentada y cinco estambres soldados en un tubo, por la parte superior. Las flores son de color amarillo-naranja (Ladner, s/f.; Waizel, 1995; Plants for the future, 2012; USDA-ARS, 2013).

En la polinización pueden intervenir insectos, pero presenta autopolinización; la floración ocurre en los meses de verano, a partir del segundo año de vida de la planta; el fruto (aquenio) es alargado, de cuatro a cinco mm, cerdoso y rematado por un vilano rubio de pelos finos, algo quebrados, constituidos por las sedas persistentes del cáliz. El rizoma

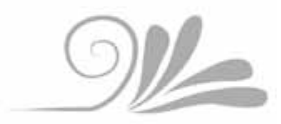

or early summer; that of the rhizomes, in early fall, when the specimen enters into latency; it must be uprooted, cleaned and chopped (Herbotecnia, n/d.).

Products for internal use are prepared as elixirs, or as homeopathic remedies in the form of globules, granules, tablets, salves, injectable solutions, drops and drinkable ampoules. Externally, it is used in poultices, mouthwashes, topic creams, elixirs, sprays, gargling solutions, gels, liniments, patches, salves, hair rinses, sticks or ointments. The dry plant is used in traditional phytotherapy (with due caution because of its toxicity) in infusions, teas, tinctures, lotions, etc. (CEDICRAT, 2009).

\section{Botanical description}

Arnica montana is an 7.87" to 23.62 " tall perennial herb with low (basal) leaves that are level with the ground; these have a bright green color, slightly downy on the upper surface, grouped in clusters (flat rosettes), ovate-ciliate and with rounded tips. The upper leaves are smaller, opposed and spear-shaped, and they are directly attached to the underground stem. From the center of the rosette rises a hairy round stem ending in one to three flower stalks, which in turn end in a head-shaped hermaphroditic inflorescence with a diameter of 7 to $8 \mathrm{~cm}$. In exceptional cases, they have one to three pairs of smaller heads on lateral branches, each made up of a series of peripheral ligulated flowers consisting of a calyx represented by fine-tipped prickly bristles, with a zygomorphic corolla formed by a layer with three teeth and an ovary ending on a forked appendix at the tip. The central flowers, at the center of the head, have a tubulous corolla or a corolla with five teeth and five stamens joined in a tube on the upper part. The flowers are orange-yellow (Ladner, n/d.; Waizel, 1995; Plants for the future, 2012; USDA-ARS, 2013).

Insects may be involved in the pollination, but self-pollination occurs; the flowering period covers the summer months, after the second year of life of the plant; the fruit (achene) is long, measures four to five $\mathrm{mm}$, is bristly and ends in a yellow pappus with fine, somewhat wavy hairs, made of the enduring silks of the calyx. The rhizome is cylindrical, erect, simple or somewhat ramified, of a dark brown color; it is generally curved and has frail, wiry rootlets on its lower surface. The diploid chromosome number of the species is 38 (Ladner, n/d.; Waizel, 1995; Plants for the future, 2012; USDA-ARS, 2013).

\section{Origin and geographical distribution}

The species is endemic in Central and Southern Europe (the Alps and the Pyrenees), in northern Spain and in southern Scandinavia. Its habitat is located at altitudes ranging between 700 and 2500 meters (the atlantica subspecies grows between 0 and $1000 \mathrm{~m}$ ); in alpine meadows or in coniferous forests in the cold or temperate mountainous regions of Europe and North America (where it has been naturalized, at the west of 
es cilíndrico, erecto, simple o algo ramificado, de color marrón oscuro, generalmente curvo y lleva frágiles raicillas nervudas en la superficie inferior. El número cromosómico diploide de la especie es 38 (Ladner, s/f.; Waizel, 1995; Plants for the future, 2012; USDA-ARS, 2013).

\section{Origen y distribución geográfica}

La especie es endémica de Europa Central y Meridional (Alpes y Pirineos), del norte de España y del sur de Escandinavia. Su hábitat está en altitudes desde los 700 hasta los 2500 metros (la subespecie atlantica crece entre 0 y 1000 m); en prados alpinos o en bosques de coníferas en las regiones montañosas frías o templadas de Europa y América del Norte len esta última área como naturalizada, en el oeste de las montañas Rocallosas, en los estados de Montana, Dakota del Norte, Dakota del Sur y Wyoming) (Kuklinski, 2000; USDA-ARS, 2013).

En Europa se distribuye en el norte (Dinamarca, Noruega y Suecia), en el centro (Austria, Bélgica, República Checa, Alemania, Hungría, Países Bajos, Polonia y Suiza), en el oriente (Bielorrusia, Lituania y Ucrania), al suroriente (Bosnia y Herzegovina, Bulgaria, Croacia, Italia, Montenegro, Rumania y Eslovenia) y en el suroeste (Francia, Portugal y España). También se introdujo y fue cultivada con éxito en Estados Unidos de América y en Escocia (Muñoz, 1987; Waizel, 1995; Kuklinski, 2000; USDA-ARS, 2013); asimismo se ha observado en México, en cuyo caso podría tratarse de plantas cultivadas (Conabio, 2011).

\section{Sinonimia científica y común}

El género Arnica pertenece a la familia Asteraceae (Compositae) y consta de 28 a 32 especies de la región templada norte, fue descrito por Carlos Linneo, con base en A. montana, que fue citada en 1753 en la obra Species Plantarum, y que ha recibido numerosos nombres científicos.

Se consideran válidos, a la fecha: Arnica alpina f. inundata Porsild; Arnica helvetica G. Don ex Loudon; Arnica lowii Holm; Arnica montana L. subsp. montana; Arnica montana L. var. montana; Arnica petiolata Schur; Cineraria cernua Thore; Doronicum montanum (L.) Lam; Doronicum oppositifolium Lam. (Font Quer, 1962; American Institute of Homeopathy, 1979; Bharatan et al., 2002; Greuter, 2006; DortaSoares, s/f.; Gotfredsen, 2009; ITIS, 2010; The Plant List, 2013; USDA-ARS, 2013; Tropicos, 2014).

Nombres considerados como inválidos: Arnica alpina Willd. ex Steud; Arnica angustifolia Turcz. ex Ledeb; Arnica plantaginifolia Gilib.; Chrysanthemum latifolium (DC.) Baksay; Doronicum arnica Desf.; Doronicum arnica Garsault; Senecio arnica E.H.L. Krause (Font Quer, 1962; American Institute of Homeopathy, the Rocky Mountains, and in the states of Montana, North Dakota, South Dakota and Wyoming) (Kuklinski, 2000; USDA-ARS, 2013).

In Europe it is distributed in the north lin Denmark, Norway and Sweden), at the center (Austria, Belgium, the Czech Republic, Germany, Hungary, the Netherlands, Poland, and Switzerland), in the east (Belorussia, Lithuania and Ukraine), in the southeast (Bosnia-Herzegovina, Bulgaria, Croatia, Italy, Montenegro, Romania, and Slovenia) and in the southwest (France, Portugal and Spain). It was also introduced and successfully cultivated in the United States of America and in Scotland (Muñoz, 1987; Waizel, 1995; Kuklinski, 2000; USDA-ARS, 2013). It has also been observed in Mexico, in which case it may have been introduced as a cultivated plant (Conabio, 2011).

\section{Scientific and common synonyms}

The genus Arnica belongs to the Asteraceae (Compositae) family and consists of 28 to 32 species of the northern temperate region; it was described by Carl Linnaeus based on A. montana, which was cited in 1753 in his work Species Plantarum and which has received a large number of scientific names.

The following are regarded as valid to date: Arnica alpina $f$. inundata Porsild; Arnica helvetica G. Don ex Loudon; Arnica lowii Holm; Arnica montana L. subsp. montana; Arnica montana L. var. montana; Arnica petiolata Schur; Cineraria cernua Thore; Doronicum montanum (L.) Lam; Doronicum oppositifolium Lam. (Font Quer, 1962; American Institute of Homeopathy, 1979; Bharatan et al., 2002; Greuter, 2006; DortaSoares, n/d.; Gotfredsen, 2009; ITIS, 2010; The Plant List, 2013; USDAARS, 2013; Tropicos, 2014).

Names regarded as invalid are: Arnica alpina Willd. ex Steud; Arnica angustifolia Turcz. ex Ledeb.; Arnica plantaginifolia Gilib.; Chrysanthemum latifolium (DC.) Baksay; Doronicum arnica Desf.; Doronicum arnica Garsault; Senecio arnica E.H.L. Krause (Font Quer, 1962; American Institute of Homeopathy, 1979; Bharatan et al., 2002; Greuter, 2006; DortaSoares, n/d.; Gotfredsen, 2009; ITIS, 2010; The Plant List, 2013; USDA-ARS, 2013; Tropicos, 2014).

Pre-Linnaean or historical names include: Alisma, Caltha alpine, Nardus celtica altera, Panacea lapsorum and Ptarmica. (The Plant List, 2013; USDA-ARS, 2013; De Boer, in Ladner, n/d.; Dorta Soares, n/d.; Font Ouer, 1962; American Institute of Homeopathy, 1979; Bharatan, 2002; Greuter, 2006; Dorta Soares, n/d.; Gotfredsen, 2009; ITIS, 2010; The Plant List, 2013; USDA-ARS, 2013; Tropicos, 2014).

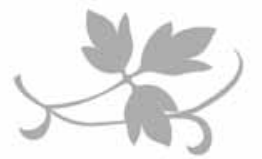


1979; Bharatan et al., 2002; Greuter, 2006; DortaSoares, s/f.; Gotfredsen, 2009; ITIS, 2010; The Plant List, 2013; USDA-ARS, 2013; Tropicos, 2014).

Denominaciones pre linneanas o históricas: Alisma, Caltha alpine, Nardus celtica altera, Panacea lapsorum y Ptarmica. (The Plant List, 2013; USDA-ARS, 2013; De Boer en Ladner, s/f;; Dorta Soares, s/f.; Font Quer, 1962; American Institute of Homeopathy, 1979; Bharatan, 2002; Greuter, 2006; Dorta Soares, s/f.; Gotfredsen, 2009; ITIS, 2010; The Plant List, 2013; USDA-ARS, 2013; Tropicos, 2014).

\section{Denominación popular}

Dalechamps adoptó el nombre "arnica" en el siglo XVI, que puede provenir del griego arnakis, que significa "abrigo de cordero" o "piel de cordero", en referencia a sus hojas suaves y vellosas, así como a los sépalos cubiertos de pelos suaves (similares al fieltro) que rodean a la flor. También es probable que provenga de ptarmica, que se refiere al estornudo que provoca su olor; montana es el epíteto geográfico e indica su localización en las montañas (Goldenmap, s/f.; Hausen y Oestman, 1988; Obón et al., 2012).

A. montana recibe distintas denominaciones según la lengua, a continuación se listan los comúnmente citados en la literatura (American Institute of Homeopathy, 1979; Muñoz, 1987; Gotfredsen, 2009; Greuter, 2006; USDA-ARS, 2013).

Alemán: Arnika, berg-wohlverleih, echte arnica y wohl verleich Checoeslovaco: Arnika horská, prha arnica y prha chlumní

Chino: Shan jin che

Danés: Arnica, arnica, arnikablomst, Almindelig guldblomme, Bierg-guldblomme, Biergvolverlej, Gammelmand y Volverlejblomst

Eslovaco: Arnika horská, brdnia y navadna arnika Español lincluye Aragonés, Asturiano, Castellano, Catalán, Galiciano, Valenciano y Vasco): Árnica, arnica, Arnicón, arnika, árnika, dorónico de Alemania, estabaco, esternudera, estornudadera, flor de tabaco, herba capital, hèrba capital, herba cheirenta, herba da papeira, herba de buitre, herba de les caigudes, hierba de las caídas, hierba santa, quina dos pobres, tabac de muntanya, tabac de pastor, tabaco, tabaco borde, tabaco de la montaña, tabac de montanya, tabaco de montaña, tabaco de monte, tabaco del diablo, talpa, talpica, usin-belar, yerba de las caídas y zebadilla. Esperanto: Arniko monta

Finlandés: Arnikki, etelänarnikki y telänarnikki

Francés: Arnica des montagnes, arnique, bétoine des montagnes, doronic des Vosges, herbe aux chutes, herbe aux prêcheurs, panacée des chutes, quinquina des pauvres, souci des Alpes, tabac des Savoyards y tabac des Vosges

Groenlandés: Sungorsiusaq

Holandés: Valkruid, valkruid sort y wolverlei

Húngaro: Árnika y hegyi árnika
Denominaciones pre linneanas o históricas: Alisma, Caltha alpine, Nardus celtica altera, Panacea lapsorum y Ptarmica. (The Plant List, 2013; USDA-ARS, 2013), (De Boer, H. En: Ladner, J. s/f.; Dorta Soares, s/f.; Font Quer, 1962; American Institute of Homeopathy, 1979; Bharatan, 2002; Greuter, 2006; Dorta Soares, s/f.; Gotfredsen, 2009; ITIS, 2010; The Plant List, 2013; USDA-ARS, 2013; Tropicos, 2014).

\section{Popular designation}

In the XV|th century, Dalechamps adopted the name "arnica", which may be derived from the Greek arnakis, meaning "sheep coat" or "sheepskin" -a reference to its soft, downy leaves, as well as to the sepals covered with soft hairs (resembling felt) that surround the flower. It may also come from ptarmica, which refers to the sneezing caused by its scent; montana is the geographical epithet that indicates that it grows on the mountains (Goldenmap, s/f; Hausen and Oestman, 1988; Obón et al., 2012).

A. montana is given different names according to the language; below is a list of the names most commonly cited in the literature (American Institute of Homeopathy, 1979; Muñoz, 1987; Gotfredsen, 2009; Greuter, 2006; USDA-ARS, 2013).

Chinese: Shan jin che

Czech: Arnika horská, prha arnica and prha chlumní

Danish: Arnica, arnica, arnikablomst, Almindelig guldblomme, Bierg-guldblomme, Biergvolverlej, Gammelmand and Volverleiblomst

Dutch: Valkruid, valkruid sort and wolverlei

Esperanto: Arniko monta

Finnish: Arnikki, etelänarnikki and telänarnikki

French: Arnica des montagnes, arnique, bétoine des montagnes, doronic des Vosges, herbe aux chutes, herbe aux prêcheurs, panacée des chutes, quinquina des pauvres, souci des Alpes, tabac des Savoyards and tabac des Vosges

German: Arnika, Berg-Wohlverleih, Echte Arnika and Wohl Verleich

Greenlandic: Sungorsiusaq

Hungarian: Árnika and hegyi árnika

English: Arnica, Celtic nard, cure all, European arnica, fallherb, golden fleece, lambskin, leopard's bane, mountain arnica, mountain daisy, mountain-tobacco, sneezewort, tumblers and wolfss bane

Icelandic: Fiallagullblóm

Italian: Arnica and tabacco di montagna

Lithuanian: Kalniné arnika

Norwegian: Solblom, Gullblom, Hestesoleie, Jonsokblom, Slåtteblom, Slåttermann, Snusblad and Tobakksblom Polish: Arnika, arnika górska, arnica pospolita and pomornik Portuguese: Arnica vulgar, arnica-da-montanha, betónica-dossaboianos, cravo-dos-Alpes, dórico-da-Alemanha, panaceiadas-queda, quina-dos-pobres, tabaco-dos-saboianos, 
Inglés: Arnica, Celtic nard, cure all, European arnica, fallherb, golden fleece, lambskin, leopard's bane, mountain arnica, mountain daisy, mountain-tobacco, sneezewort, tumblers y wolf's bane

Islándico: Fiallagullblóm

Italiano: Arnica y tabacco di montagna

Lituano: Kalniné arnika

Norvego: Solblom, Gullblom, Hestesoleie, Jonsokblom, Slåtteblom, Slåttermann, Snusblad y Tobakksblom

Polaco: Arnika, arnika górska, arnica pospolita y pomornik

Portugués: Arnica vulgar, arnica-da-montanha, betónica-dossaboianos, cravo-dos-Alpes, dórico-da-Alemanha, panaceiadas-queda, quina-dos-pobres, tabaco-dos-saboianos, tanchagem-dos-Alpes y tabaco-dos-Vosgos

Ruso: Арника горная

Sueco: Arnika, hästfibbla, hästfibla y slåttergubbe

Turco: Altın Çiçek, arnika y Öküzgözüotu

Ucraniano: Арніка гірська

La primera vez que se menciona "árnica" fue en el siglo XII y lo hizo Santa Hildegarda, esta denominación popular fue desconocida entre los clásicos griegos y romanos, y tampoco está citada en los primeros textos medievales. Al parecer fue hasta el siglo XVI que se descubrieron sus poderes medicinales (Opsomer, 1969, citado por Obón et al., 2012).

El árnica es un antiguo tratamiento europeo para contusiones, esguinces y dolores musculares, molida y aplicada sobre cualquier herida que necesitara sanación. En la Alemania temprana utilizaban todas las partes de la planta como remedios populares; los botánicos, Matthiolus, Gesner y Clusius tenían conocimiento de sus cualidades curativas, ya que eran usadas por la gente común, de hecho, las primeras ilustraciones de la especie (con el nombre de alisma) aparecen en el libro de herbolaria que escribió Matthiolus (Schar, 2010).

En el siglo XVI Franz Joel la recomendó y en 1625 Johann Jakob von Bergzabern (conocido como Taberna montanus) utilizó A. montana para tratar contusiones y hematomas, además divulgó estas propiedades en su libro de herbolaria, en el que aparece el comentario: "called arnica by the Medici". En 1678 se usó como alternativa a la "quina" para tratar la fiebre y Collin de Viena verificó que mil pacientes del Hospital Pazman se curaron de fiebres intermitentes cuando se les administraron flores de A. montana, esta fue reconocida en la Farmacopea de Londres en 1788 y después cayó en desuso; actualmente se utiliza y tiene una posición importante para curar esguinces y contusiones aplicándola en forma de tintura (Schar, 2010).

\section{Usos medicinales}

Auxiliar en el tratamiento de: la abrasión de la piel, amigdalitis, ampollas no abiertas, anemia, anginas, apopleija, artralgia,
tanchagem-dos-Alpes and tabaco-dos-Vosgos

Russian: Арника горная

Slovak: Arnika horská, brdnia and navadna arnika Spanish (includes Aragonian, Asturian, Castillian, Catalonian, Gallician, Valencian, and Basque): Árnica, arnica, Arnicón, arnika, árnika, dorónico de Alemania, estabaco, esternudera, estornudadera, flor de tabaco, herba capital, hèrba capital, herba cheirenta, herba da papeira, herba de buitre, herba de les caigudes, hierba de las caídas, hierba santa, quina dos pobres, tabac de muntanya, tabac de pastor, tabaco, tabaco borde, tabaco de la montaña, tabac de montanya, tabaco de montaña, tabaco de monte, tabaco del diablo, talpa, talpica, usin-belar, yerba de las caídas and zebadilla.

Swedish: Arnika, hästfibbla, hästfibla and slåttergubbe

Turkish: Altın Çiçek, arnika and Öküzgözüotu

Ukrainian: Арника горная

The first mention of "arnica" was made by Saint Hildegard in the X $\|^{\text {th }}$ century; this popular name was unknown to the classic Greeks and Romans and is not cited in the first medieval texts (Opsomer, 1969, cited by Obón et al., 2012).

Arnica is an ancient European treatment for contusion, sprains, and muscular pain, which was ground and applied on any wound that might require healing. In early Germany, every part of the plant was used in popular remedies; the botanists Matthioulus, Gesner and Clusius had knowledge of its healing qualities, since the plant was used by the common people; actually, the first illustrations of the species (under the name alisma) appear in Matthioulus' work on medicinal herbs (Schar, 2010).

In the $\left.X V\right|^{\text {th }}$ century, Franz Joel recommended it, and in 1625 Johann Jakob von Bertgzabern (known as Taberna montanus) used $A$. montana to treat contusions and hematomas; furthermore, he disseminated these properties in his book on medicinal herbs, in which the comment "called arnica by the Medici" appears. In 1678 it was used as an alternative to "quinine" for treating fever, and Collin of Vienna verified that one thousand patients of the Pazman Hospital were healed from intermittent fevers when they were administered $A$. montana flowers. This species was acknowledged in the 1788 edition of the London Pharmacopoeia and afterwards fell into disuse. Today its use has been reinstated, and it plays a prominent role, as a tincture, in the treatment of sprains and contusion (Schar, 2010).

\section{Medicinal uses}

It is an aid in the treatment of: skin abrasion, tonsillitis, unburst blisters, anemia, quinsy, arthralgia, arthritis, arteriosclerosis, asthma, atony of the heart, blood tonic, bed-wetting lurinating in bed), bites, bleeding, blows, brain concussion, bronchitis, bumps, burns, cachexia, car or sea sickness, cardiovascular diseases, circulatory problems, clots in the blood vessels, 
artritis, asma, ateroesclerosis, atonía cardiaca, bronquitis, caquexia, chichones, coágulos en los vasos sanguíneos, congestión, conmociones cerebrales, contusión, curación de heridas, debilidad nerviosa, deficiente circulación sanguínea, deprutativo, desgarres, distensiones musculares, diarrea, disfonía, dislocaciones, dispepsia, dolor al correr, dolor de garganta, dolor muscular, dolor reumático, eczema, enfermedades estomacales, cardiovasculares, de la columna vertebral, del hígado y oculares, equimosis, esguinces, faringitis, fiegre, gota, hematomas, hematomas resultantes de choques o golpes, heridas, hipertensión, hipotensión, ictericia, inflamación, inflamación de la boca, inflamación de las mucosas bucales, influenza, llagas bucales, llagas persistentes, malaria, malestares reumáticos, mareo, mareos al viajar en auto o en transportes marítimos, mojar la cama (orinar en ella), mordeduras, neuralgia, parálisis, pérdida de cabello, periodontitis, picor vaginal, piorrea dental, podagra, problemas cardiacos, circulatorios o de las encías, golpes, quemaduras, resfriado común, reumatismo, sangrado, síncope, tos, tosferina, trastornos vasculares o venosos, tumores, úlceras rebeldes y várices (Font-Quer, 1962; Saury, 2004; Gotfredsen, 2009; Botanical, 2014).

Se recomienda como afrodisiaco, analgésico, anticaspa, anticatarral, anticonvulsionante antidematosis, antiespasmódico, antiinflamatorio, amtimicrobiano, antiséptico, diurético, estimulante, estimulante de la circulación sanguínea, inductor de la sudoración, en solución para el lavado de ojos, limitador de infecciones, polvo estornudatorio, reductor de inflamaciones, tónico y ungüento oftálmico (Font-@uer, 1962; Saury, 2004; Gotfredsen, 2009; Botanical, 2014).

\section{El árnica en la homeopatía}

Se le considera un policresto; es decir que tiene múltiples usos o cura muchas enfermedades. Samuel Hahnemann en Fragmenta ( 1805) y Materia Médica Pura (1811-31) menciona las aplicaciones medicinales, especialmente en padecimientos como la epilepsia y los mareos (American Institute of Homeopathy, 1979). Su empleo en homeopatía se basa en su poder para iniciar la reparación de los tejidos a nivel celular en los sitios donde se experimenta un trauma físico agudo, en estos casos se sugiere utilizar el árnica lo antes posible para ayudar a reducir la hinchazón, los moretones y para reparar el daño de tejido muscular, así como de la piel. También promueve la curación de los músculos sometidos a un exceso de trabajo, por lo cual se debe aplicar antes y después de actividades deportivas, cirugías o tratamientos dentales (Historical Remedies, 2012). Se usa como auxiliar para curar ateroesclerosis, debilidad cardiaca, hemorragias y venas inflamadas, entre otras enfermedades (American Institute of Homeopathy, 1979; Lampe y McCann, 1985; Kuklinski, 2000; Seror, 2001; Gotfredsen, 2009; USDAARS, 2013; Morrell, s/f.). common cold, congestion, contusion, cough, muscular distension, diarrhea, dysphonia, dislocations, dyspepsia, ecchymosis, eczema, faringitis, fever, gout, gum disease, hair loss, healing of wounds, heart problems, hematomas, hematomas from shocks or blows, hypertension, hypotension, influenza, jaundice, liver and eye diseases, malaria, mouth ulcers, muscular pain, nervous weakness, neuralgia, paralysis, periontoditis, persistent ulcers, podagra, poor blood circulation, rheumatic ailments, rheumatic pain, rheumatism, running pain, sickness, sore throat, stomach diseases, spine ailments, sprains, swelling, swelling of the mouth, swelling of the mucosae of the mouth, syncope, tears, tumors, unmanageable ulcer, vaginal itch, varicose veins, vascular or venous disturbances, whooping cough, and wounds (Font-Quer, 1962; Saury, 2004; Gotfredsen, 2009; Botanical, 2014).

It is recommended as afrodisiac, analgesic, anti-dandruff, anti-cold, anti-convulsive, anti-edematous, anti-spasmodic, anti-inflammatory, antimicrobial, antiseptic, diuretic, stimulant, stimulant of blood circulation, sweat-inducing agent, in a solution as an eyewash, infection limiting agent, sneezing powder, inflammation reducer, tonic and eye ointment (Font-Quer, 1962; Saury, 2004; Gotfredsen, 2009; Botanical, 2014).

\section{Uses of arnica in homeopathy}

It is regarded as a polycrest remedy; i.e. it has multiple uses or heals many conditions. Samuel Hahnemann -in his works Fragmenta (1805) and Materia Medica Pura (1811-31)- mentions is medicinal applications, particularly in such ailments as epilepsy and dizziness (American Institute of Homeopathy, 1979). Its use in homeopathy is based on its power to initiate the repair of the tissues at cell level in those parts where acute physical trauma has been experienced. In these cases, it is suggested to apply arnica as soon as possible in order to help reduce the swelling and the bruises and to repair the damage to the muscular tissue, as well as to the skin. It also promotes the healing of the muscles subjected to excess work; therefore, it must be applied before and after sport activities, surgeries or dental treatments (Historical Remedies, 2012). It is used as an aid in healing arteriosclerosis, heart weakness, hemorrhage, and swollen veins, among other conditions (American Institute of Homeopathy, 1979; Lampe y McCann, 1985; Kuklinski, 2000; Seror, 2001; Gotfredsen, 2009; USDA-ARS, 2013; Morrell, n/d.).

\section{Counter-indications and secondary effects}

Arnica works as a poison for grazing mammals; it is tolerated only by goats. In the case of human beings, when administered orally and in high doses, it may cause severe headaches, miscarriage, delirium, convulsions and, occasionally, fatal poisoning la handful of leaves may be enough to bring about these effects). Its toxicity is greater when ingested, as it acts upon the liver and the central nervous system, causing vomiting, 


\section{Contraindicaciones y efectos secundarios}

El árnica funciona como veneno para los mamíferos que pastorean, solo es tolerada por las cabras y los chivos; en el caso del humano, cuando se administra por vía oral y en dosis altas, puede ocasionar fuertes dolores de cabeza, aborto, delirio, convulsiones, y en ocasiones envenenamientos mortíferos (un puñado de hojas puede ser suficiente). Su toxicidad es mayor si se ingiere, porque actúa en el hígado y el sistema nervioso central, lo que causa vómitos, cefalea, mareos y disfunción respiratoria; también afecta, la función del corazón (por sus principios cardiotónicos) y ocasiona el incremento de la presión arterial. Provoca irritación de las membranas mucosas, cuando se administra por vía oral es posible que origine gastroenterosis fatal, parálisis de los músculos voluntarios y cardiacos, incremento o disminución del pulso, palpitaciones del corazón, dificultad para respirar e incluso causar la muerte. Debe evitarse su contacto con los ojos o áreas sensibles como heridas abiertas. Su uso está contraindicando en niños o durante el embarazo y la lactancia Morton, 1971; Lampe, 1985; Hausen y Oestman, 1988; Waizel, 1995; Kuklinski, 2000; Duke y Bogenschutz-Godwin, 2002; Gotfredsen, 2009; Schar, 2010; Vogel, 2013).

Las planta contiene lactonas sesquiterpénicas (helenalina, dihidrohelenalina, arnifolina y arnicolida), las cuales tienen efectos antiinflamatorios y analgésicos que al usarse tópicamente, en dosis elevadas, producen una respuesta alergénica o dermatitis de contacto en algunos individuos; esto se ha constatado en agricultores, granieros, jardineros y floristas que están en contacto con A. montana (Morton, 1971; Lampe, 1985; Hausen y Oestman, 1988; Waizel, 1995; Kuklinski, 2000; Duke y Bogenschutz-Godwin, 2002; Gotfredsen, 2009; Schar, 2010; Vogel, 2013).

Las aplicaciones deben ser en forma de diluciones y por vía tópica para aliviar inflamación en la cabeza locasionada por un golpe), esguinces, caídas, torceduras, luxación, malestar osteo-muscular crónico, para acelerar la cicatrización de una lesión traumática o reducir dolor agudo. En la medicina popular se recomienda emplearla de manera local y sobre la piel; además de, evitar su ingesta (Morton, 1971; Lampe, 1985; Hausen y Oestman, 1988; Waizel, 1995; Kuklinski, 2000; Duke, 2002; Gotfredsen, 2009; Schar, 2010; Vogel, 2013).

\section{Fitoquímica}

Se han realizado numerosos análisis químicos de Arnica montana, en especial de las hojas, las inflorescencias y el rizoma, a partir de ellos se han logrado aislar varios compuestos orgánicos, en particular se han identificado aceites esenciales, ácido angélico, ácido baldriánico, ácido cafeico, ácido cinámico, ácido clorogénico, ácido dioxisteárico, ácido esteárico, ácido fórmico, ácido fumárico, ácido gálico, ácido isobutírico, headaches, dizziness and respiratory dysfunction. It also affects the function of the heart (due to its cardiotonic effects) and raises the blood pressure. It causes irritation of the mucous membranes; when administered orally, it may cause a fatal gastroenterosis, paralysis of the voluntary and heart muscles, or an increase or decrease of the heart rate, heart palpitations, breathing problems or even death. Its contact with the eyes or sensitive areas like open wounds must be avoided. Its use is counter-indicated in children and infants, and in pregnant and lactating women Morton, 1971; Lampe, 1985; Hausen y Oestman, 1988; Waizel, 1995; Kuklinski, 2000; Duke y Bogenschutz-Godwin, 2002; Gotfredsen, 2009; Schar, 2010; Vogel, 2013).

The plant contains sesquiterpene lactones (helenalin, dihydrohelenalin, arnifoline and arnicolide), which have anti-inflammatory and analgesic effects and which, when used topically and in high doses, produce an allergenic response or contact dermatitis in certain subjects. This has been confirmed in farmers, gardeners and florists who are in contact with $A$. montana Morton, 1971; Lampe, 1985; Hausen y Oestman, 1988; Waizel, 1995; Kuklinski, 2000; Duke y Bogenschutz-Godwin, 2002; Gotfredsen, 2009; Schar, 2010; Vogel, 2013).

The applications must be topical and in the form of dilutions in order to relieve inflammations in the head (caused by blows), strains, falls, sprains and chronic osteo-muscular discomfort, to accelerate the healing of a traumatic injury or to reduce sharp pain. In popular medicine its topical use on the skin is recommended; also, it should not be ingested (Morton, 1971; Lampe, 1985; Hausen and Oestman, 1988; Waizel, 1995; Kuklinski, 2000; Duke, 2002; Gotfredsen, 2009; Schar, 2010; Vogel, 2013).

\section{Phyłochemistry}

Numerous chemical analyses of Arnica montana have been performed, particularly of its leaves, inflorescences and rhizome. Various organic compounds have been isolated from them; in particular, essential oils, angelic acid, baldrianic acid, caffeic acid, cinnamic acid, chlorogenic acid, dioxy-stearic acid, formic acid, fumaric acid, gallic acid, isobutyric acid, isovaleric acid, lactic acid, lauric acid, malic acid, palmitic acid, silicic acid, succinic acid, tanic acid, valerianic acid, albumina, alkaloid, camphor, arnicin, arnicolide, arnilenediol, arnifolin, arnisterol, astragalin, reductive sugars, betain, beta-lactucerol, beta-sitosterol, betuletol, cadinene, caempherol, carnaubylalcohol, carotene, carotenoids, catechol tannins, wax, choline, coumarin, Dien-tetraine, dihydrohelenalin, scopoletin, bitter essence, spinacetin, sterols, eupafoline, faradiol, phytosterols, flavo-glucoside, flavones, flavonoids, fructose, glucoside, gum,

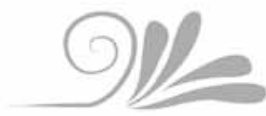


ácido isovalérico, ácido láctico, ácido láurico, ácido málico, ácido palmítico, ácido silíicico, ácido succínico, ácido tánico, ácido valeriánico, albúmina, alcaloide, alcanfor, arnicina, arnicolida, arnilenediol, arnifolina, arnisterol, astragalina, azúcares reductores, betaína, Beta-lactucerol, Beta sitoserol, betuletol, cadineno, caempferol, carnaubilalcohol, caroteno, carotenoides, catecol-taninos, cera, colina, cumarina, Dien-tetrain, dihidrohelenalina, escopoletina, esencia amarga, espinacetina, esteroles, eupafolina, faradiol, fitosteroles, flavo-glucósido, flavonas, flavonoides, fructosa, glucósido, goma, helenalina, helenien, hispidulina, humuleno, inulina, iridictiol, isoquercitina, quercetina, laciniatina, lactonas sesquiterpénicas, loliólido, luteína, luteolina, mirceno, mucílago, poliacetilenos, polisacáridos, procianidina, quercetagetina, quercetina, resina, sacarosa, taninos, taraxastano, taraxasterol, timol, timol-metil éter, tricadien-tetraina, tridecapentaina, tridecen-1-pentaina, trimetilamina, triterpenos, umbelliferona, xantofila, xantofila-epóxido y zeaxantina (Duke, 1998; Kuklinski, 2000; Gotfredsen, 2009; Ladner, s/f.).

\section{Importancia económica}

Arnica montana tiene usos importantes como planta de ornato y en la jardinería; sin embargo, se utiliza para la elaboración de numerosos productos empleados en cosmética y perfumería, como aditivos o saborizantes en la preparación de bebidas alcohólicas y en la medicina humana y veterinaria. Actualmente es uno de los remedios naturales tópicos más populares (CEDICRAT, 2009).

En el mercado alemán se ofrecen cerca de 300 preparaciones medicinales que contienen extracto de árnica y en Canadá son aproximadamente 20. En España, en 2009 se contabilizaron 69 empresas que comercializan 171 productos, del total la materia prima $24 \%$ corresponde a la flor seca y su extracto, mientras que $76 \%$ a los productos finales elaborados (CEDICRAT, 2009; Agriculture and Agri-Food Canada, 2012).

A nivel mundial se estima que en 1998 se elaboraron más de 100 preparaciones farmacéuticas que utilizaron $50000 \mathrm{~kg}$ de flores secas de A. montana lequivalente a 250000 a $300000 \mathrm{~kg}$ de flores frescas), así como cientos de kilogramos de rizomas. CEDICRAT (2009) cita aproximadamente 300 preparaciones, con datos de Alemania y de 20 productos canadienses (Agriculture and Agri-Food Canada, 2012).

El precio pagado por kilogramo de flor seca de A. montana varía de 15 a $30 €$. La calidad medicinal está determinada por la Farmacopea Europea y se expresa en un mínimo de $0.40 \% \mathrm{~m} \mathrm{~m}^{-1}$ de lactonas sesquiterpénicas totales, referidas como tiglato de dihidrohelenalina en el medicamento desecado (CEDICRAT, 2009).

\section{El cultivo de la especie y países productores}

Dado que A. montana ha sido sujeta de sobreexplotación hoy helenalin, helenien, hyspidulin, humulene, inulin, eriodictiol, isoquercetin, quercetin, laciniatin, sesquiterpene lactones, loliolide, lutein, luteolin, mircene, mucilage, polyacetylenes, polysaccharides, procyanidin, quercetagetin, quercetin, resin, saccharose, tanins, taraxastane, taraxasterol, tymol, tymol-methyl ether, tri cadeine-tetraine, tri decca pentaine, trimethylamine, triterpenes, umbelliferone, xantophyl, and zeaxanthin (Duke, 1998; Kuklinski, 2000; Gotfredsen, 2009; Ladner, n/d.).

\section{Economic relevance}

Arnica montana has important uses as an ornamental plant and in gardening. However, it is used for making a large number of products used as cosmetics and in perfumes, as additives or flavorings, for making alcoholic beverages and in human and veterinary medicine. Today it is one of the most popular topical natural remedies (CEDICRAT, 2009).

Nearly 300 medicinal preparations containing arnica extracts are sold in Germany, and approximately 20 in Canada. In 2009, 69 firms selling 171 arnica products were counted in Spain; 24 \% of these products are raw materials (dried flowers and extract), and $76 \%$, final manufactured products (CEDICRAT, 2009; Agriculture and Agri-Food Canada, 2012).

At world level, it is estimated that in 1998 more than 100 pharmaceutical preparations were made, using $50000 \mathrm{~kg}$ of A. montana dried flowers lequivalent to 250000 to $300000 \mathrm{~kg}$ of fresh flowers), as well as hundreds of kilograms of rhizomes. CEDICRAFT (2009) cites approximately 300 preparations, with data for Germany and for 20 Canadian products (Agriculture and Agri-Food Canada, 2012).

The price paid per kilogram of dried A. montana flowers ranges between 15 and $30 €$. The medicinal quality is determined by the European Pharmacopoeia and is expressed in a minimum of $0.40 \% \mathrm{~m} \mathrm{~m}^{-1}$ of total sesquiterpene lactones, referred to as dihydro-henelanin tiglate in the dessicated medicine (CEDICRAT, 2009).

\section{The cultivation of the species and producing countries}

Since A. montana has been subjected to overexploitation, today it is under strict protection; it is included in the IUCN Red List of Threatened Species, as well as in the red data books and lists of many European countries. This reflects the efforts to preserve it and to prevent the reduction of its wild populations (Phillips, n/d.; CEDICRAT, 2009; Sugier, et al., 2013). In Germany it is cultivated for use as a herbal remedy, and other European countries are analyzing the possibility of developing and implementing the general guidelines to carry out sustainable collections of indigenous medicinal and aromatic plants; some examples are taking only half of the specimens in bloom, collecting a single capitulum per specimen 
está bajo estricta protección, se incluye en la Lista Roja de Especies Amenazadas (IUCN), así como en los libros y listas de datos rojos de muchos países europeos. Esto es el reflejo de los esfuerzos cuyo fin es su conservación y evitar la diminución de las poblaciones silvestres (Phillips, s/f.; CEDICRAT, 2009; Sugier, et al., 2013). En Alemania se cultiva para utilizarla como remedio herbolario y se está analizando la posibilidad de que otros países europeos desarrollen e implementen las directrices generales para realizar recolecciones sostenibles de plantas medicinales y aromáticas indígenas; algunos ejemplos incluyen solo tomar la mitad de los individuos con flor, colectar un solo capítulo de flor por ejemplar y promover la propagación con fines industriales (Muñoz, 1987; [Traffic, 1998 y EMEA, ambos citados en Ladner, s/f.]; CEDICRAT, 2009; Medicine Hunter, 2012; Phillips, 2013; Botanical, 2014; Herbotecnia, s/f; Sugier et al., 2013).

Alemania es el principal importador de árnica, tradicionalmente, se ha obtenido de los Alpes aunque en la actualidad los principales proveedores son España, India, Italia, Rumania, Rusia, Suiza y la ex Yugoslavia. Hay plantaciones importantes en Escocia, Estonia, Francia, Hungría e Inglaterra; asimismo se han realizado ensayos para introducirla en zonas de Chile con suelos pobres y en altitudes mayores a $1000 \mathrm{~m}$.

A. montana requiere de un cuidadoso cultivo en suelo con $\mathrm{pH}$ ácido (4 a 6) o neutro, granítico, sin cal, con poco contenido de nitrógeno y bajo nivel de nutrientes, rico en silice, humus y humedad constante. La planta puede crecer al sol, pero prefiere sitios ligeramente sombreados y clima templado o frío, húmedo y lluvioso; en España llega a desarrollarse en pantanos y ciénagas. No resiste agua calcárea y es muy sensible a la contaminación ambiental, en especial a los compuestos nitrogenados (Muñoz, 1987; [Traffic, 1998 y EMEA, ambos citados en Ladner, s/f.]; CEDICRAT, 2009; Medicine Hunter, 2012; Phillips, 2013; Botanical, 2014; Herbotecnia, s/ff; Sugier et al., 2013).

Su reproducción es posible mediante la siembra de semillas en almácigos inoculados con hongos micorrízicos y su posterior transplante, o bien a partir de los rizomas, el cual es más efectivo, ya que el porcentaje de germinación es bajo. La especie puede ser atacada por mohos y diferentes especies de insectos; en España las larvas de moscas (Tephritis arnicae Linnaeaus, 1758 y Trypeta arnicivora Loew, 1844), infestan con frecuencia el receptáculo floral, por ello, se sugiere recolectar las flores en cuanto abren para evitar la depredación y la destrucción de los frutos (Muñoz, 1987; [Traffic, 1998 y EMEA, ambos citados en Ladner, s/f.]; CEDICRAT, 2009; Medicine Hunter, 2012; Phillips, s/f.; Botanical, 2014; Herbotecnia, s/f.; Sugier et al., 2013).

El precio del árnica como materia prima es atractivo, lo cual justifica que se realicen investigaciones para su propagación en México, para consumo local o para exportación; por lo anterior, es recomendable realizar estudios de mercado y and promoting the spread of the species for industrial purposes (Muñoz, 1987; : Traffic, 1998 and EMEA, both citated by Ladner, n/d.]; CEDICRAT, 2009; Medicine Hunter, 2012; Phillips, 2013; Botanical, 2014; Herbotecnia, n/d.; Sugier et al., 2013).

Germany is the main importer of arnica. Traditionally, this plant has been obtained from the Alps, although today the main suppliers are Spain, India, Italy, Romania, Russia, Switzerland and the former Yugoslavia. There are important plantations in Scotland, Estonia, France, Hungary and England; likewise, assays have been made to introduce it into certain areas of Chile with poor soils and at altitudes above $1000 \mathrm{~m}$.

A. montana requires a careful cultivation on soils with an acid (4-6) or neutral pH that are granitic, lime-free, with a low content of nitrogen and a low level of nutrients, rich in silica, humus and constantly moist. The plant can grow under the sun, but it prefers slightly shady places and a temperate or cold, humid and rainy weather. In Spain, it develops even in marshes and swamps. It does not resist calcareous water and it is very sensitive to environmental pollution, especially to nitrogenated compounds (Muñoz, 1987; [Traffic, 1998 and EMEA, both cited by Ladner, n/d.]; CEDICRAT, 2009; Medicine Hunter, 2012; Phillips, 2013.; Botanical, 2014; Herbotecnia, n/d.; Sugier et al., 2013).

It can be reproduced by planting the seeds in seedbeds inoculated with mycorrhizal fungi and later transplanting them, or from the rhizomes; the latter method is more effective, since it has a low germination rate. The species may be attacked by mildews and by various species of insects. In Spain, fly larvae (Tephritis arnicae Linnaeaus, 1758 and Trypeta arnicivora Loew, 1844) often infest the flower receptacle; for this reason, it is recommended to collect the flowers as soon as the plant blooms, in order to avoid its depredation and the destruction of its fruits frutos (Muñoz, 1987; [Traffic, 1998 and EMEA, both citated by Ladner, n/d.]; CEDICRAT, 2009; Medicine Hunter, 2012; Phillips, n/d.; Botanical, 2014; Herbotecnia, n/d.; Sugier et al., 2013).

The price of Arnica as a raw material is attractive; this warrants carrying out research in order to spread the species in Mexico for local consumption or exportation. For this reason, further market studies and small-scale agronomical assays are recommended to ensure its successful introduction and cultivation. We should highlight that the country has suitable soils for its development. 39 companies using or supplying Arnica montana for the production of cosmetics or drugs or selling it within Mexico have been registered (QuimNet.com, 2014).

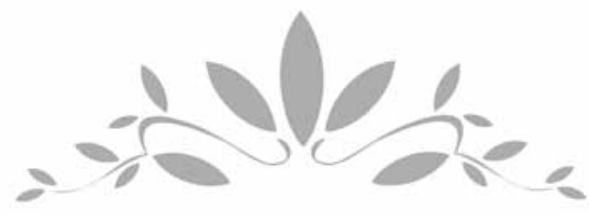


ensayos agronómicos a pequeña escala que permitan asegurar el éxito de su introducción y cultivo; es de resaltar que en el país hay suelos adecuados para su desarrollo. Se han registrado 39 empresas que emplean o proveen Arnica montana para la producción de cosméticos o medicamentos, o que la comercian en la república mexicana (QuimNet.com, 2014).

\section{Agradecimientos}

A la Comisión de Operación y Fomento de Actividades Académicas (COFAA) y al Programa de Estímulos al Desempeño Docente, ambos del Instituto Politécnico Nacional (IPN). Al Dr. Juan Salas-Benito y a los revisores de la contribución por sus comentarios.

\section{Referencias}

Agriculture and Agri-Food Canada. 2012. Arnica species (Arnica). http://www. agr.gc.ca/eng/science-and-innovation/science-publications-andresources/resources/canadian-medicinal-crops/medicinal-crops/ arnica-species-arnica/?id= 1300900418443 (27 de enero de 2014).

American Institute of Homeopathy. 1979. The Homeopathic Pharmacopoeia of the United States. American Institute of Homeopathy. Fall Church's, VA, USA. $721 \mathrm{p}$.

Bharatan, V., J. C. Humphries and R. J. Barnett. 2002. Plant Names in Homeopathy. The Natural History Museum. London, UK. 126 p.

Botanical. 2014. Propiedades del Árnica. In: El mundo de las Plantas. Botanical-online SL. http://www.botanical-online.com/ medicinalsarnica.htm (27 de enero de 2014).

Centre de Desenvolupament Rural Integrat de Catalunya (CEDICRAT). 2009. Uso sin abuso. Producción sostenible de flor de árnica en el Pirineo. Centre de Desenvolupament Rural Integrat de Cataluyna. http:// www.ciencias-marinas.uvigo.es/bibliografia _ ambiental/plantas/ Arrnica\%20montana.pdf (23 de enero de 20ī4).

Comisión Nacional para el Conocimiento y Uso de la Biodiversidad (CONABIO). 2011. La Biodiversidad en Puebla: Estudio de Estado. México. Comisión Nacional para el Conocimiento y Uso de la Biodiversidad, Gobierno del Estado de Puebla, Benemérita Universidad Autónoma de Puebla. Puebla, Pue., México. 440 p.

De Boer, H. s/f. Arnica montana synonyms. In: Ladner, J. (ed.). Arnica montana L. Grassland species. Profiles. CIAT/FAO http://www.fao.org/ag/AGP/ AGPC/doc/Gbase/data/pf000462.htm (3 de noviembre de 2013)

Díaz, J. L. 1976. Índice y Sinonimia de las Plantas Medicinales de México. Instituto Mexicano para el Estudio de las Plantas Medicinales (IMPEPLAM). México, D.F., México. 358 p.

Dorta Soares, A. A. s/f. Estudo comparativo das matérias-primas de origem vegetal utilizadas em Homeopatia em farmacopéias homeopáticas. http://lamasson.com.br/biblioteca/biblioteca/pesquisahomeopatica/ artigogigio.htm (5 de abril de 2013).

Duke, A. J. 1998. Chemicals in: Arnica montana L. (Asteraceae) -- Leopard'sBane, Mountain Tobacco. In: Dr. Duke's. Phytochemical and Ethnobotanical Databases. http://www.ars-grin.gov/cgi-bin/duke/ farmacy2.pl (2 de noviembre de 2013).

Duke, A. J. and J. M. Bogenschutz-Godwin. 2002. Handbook of Medicinal Herbs. Second Ed. CRC Press. Boca Raton, FL, USA. 896 p.

Font-Quer, P. 1962. Plantas Medicinales. El Dioscórides Renovado. Editorial Labor. Barcelona, España. 1033 p.

Goldenmap. s/f. Arnica montana. http://es.goldenmap.com/Arnica _ montana (21 de enero de 2014).

Gotfredsen, E. 2009. Liber Herbarium II: The incomplete reference-guide to herbal medicine. http://www.liberherbarum.com/Pnl221.HTM (2 de noviembre de 2013).

Greuter, W. 2006. Compositae (pro parte majore). In: Euro+Med. 2006. Euro+Med PlantBase - the information resource for Euro-Mediterranean plant diversity. http://ww2.bgbm.org/EuroPlus/Med/ (1 de noviembre de 2013).

\section{Acknowledgments}

The authors wish to express our gratitude to the Comisión de Operación y Fomento de Actividades Académicas (COFAA) and the Programa de Estímulo al Desempeño Docente of the Instituto Politécnico Nacional (IPN), as well as to Dr. Juan Salas-Benito and to the reviewers of this paper for their comments.

End of the English versio

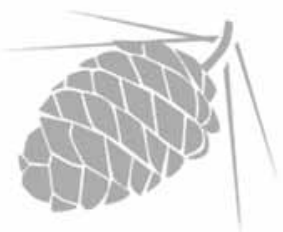

Hausen, B. M. and G. Oestman. 1988. The incidence of occupationally induced allergic skin diseases in a large flower market. Derm-Baruf-Umwelt. 36(4): 117- 124

Herbotecnia. s/f. Árnica europea. http://www.herbotecnia.com.ar/exo-arnica-m. html (26 de enero de 2014).

Historical Remedies. 2012. Arnica. http://www.historicalremedies.com/arnica (10 de noviembre de 2013).

Integrated Taxonomic Information System (ITIS). 2010. The Integrated Taxonomic Information System. Catalogue of Life: 2010 Annual Checklist. http:// www.catalogueoflife.org/annual-checklist/2010/info/about (14 de enero de 2014).

Kuklinski, C. 2000. Farmacognosia. Estudio de las drogas y sustancias medicamentosas de origen natural. Ediciones Omega. Barcelona, España. 528 p.

Ladner, J. n.d. Arnica montana L. Grassland species. Profiles. http://www.fao.org/ ag/AGP/AGPC/doc/Gbase/data/pf000462.htm (3 de noviembre de 2013).

Lampe, K. G. and A. M. McCann. 1985. AMA Handbook of poisonous and injurious plants. Cited In USDA, ARS. National Genetic Resources Program. Germplasm Resources Information Network-(GRIN) [Online Database]. National Germplasm Resources Laboratory. http://www.ars-grin.gov/cgi-bin/npgs/htm//stdlit.pl?Lampe\%20\&\%20 McCann (1 de noviembre de 2013).

Martínez, M. 1979. Catálogo de Nombres Vulgares y Científicos de Plantas Mexicanas. Edición del Fondo de Cultura Económica. México, D.F., México. $1220 \mathrm{p}$.

Medicine Hunter. 2012. Arnica. http://www.medicinehunter.com/arnica\#"sthash. poCExSua.dpuf ( 11 de noviembre de 2013).

Morton, F. J. 1971. Major medicinal plants. Charles C. Thomas Publ. Springfield, MA, USA. 288 p.

Morrell, P. n.d. Hahnemann's First Proving's. http://homeoint.org/morrell/articles/ firstprovings.htm ( 11 de noviembre de 2013).

Muñoz, F. 1987. Plantas medicinales y aromáticas. Estudio, Cultivo y Procesado. Mundi-Prensa Libros. Madrid, España. 369 p.

Nowack, T. 2002. Lowland arnica - culivation in gostyńskoleszczyński region. Wydawnictwa Polskiego Komitetu Zielarskiego 1: 18- 19.

Obón, C., D. Rivera, A. Verde, J. Fajardo, A. Valdés, F. Alcaraz, y M. A. Carvalho. 2012. Arnica: A multivariate analysis of the botany and ethnopharmacology of a medicinal plant complex in the Iberian Peninsula and the Balearic Islands. Journal of Ethnopharmacology. 144(1): 44-56.

Phillips, B. 2013. the Book of Herbs. An illustrated A-Z of the world's most popular culinary and medicinal plants. Cedar Fort, Inc. http://books. google.es/books?id=KGlhT29atfkC\&pg=PT151 \&dq=Phillips,+B.++the+ Book+of+Herbs.\&hl=es\&sa=X\&ei=fOk $\quad$ VK3CFciV8gGJwYGoCA\& ved=OCDMOuwUwAA\#v=onepage\&q=Phillips\%2C\%20B.\%20\%20 the\%2OBook\%20 of\%2OHerbs.\&f=false ( 15 de octubre de 2014). 
Plants for a Future. 2012. Arnica http://www.pfaf.org/user/Plant. aspx? LatinName=Arnica+montana ( 11 de noviembre de 2013).

QuimNet.com. 2014. Información y Negocios segundo a segundo http://www. eindustria.com/principal/resultados _ busqueda.php?d=P\&N=arnica (11 de noviembre de 2013).

Saury, A. 2004. Las Plantas Fumables Usos y Propiedades. Mandala Ediciones. Madrid, España. 99 p.

Schar, D. 2010. Arnica montana http://doctorschar.com/archives/mountainarnica-montana/ (11 de noviembre de 2013).

Seror, R. 2001. Pathogénésies réalisées par le Dr Samuel Christian Frédéric Hahnemann, sa famille et ses élèves. Arnica Montana. http:// homeoint.org/seror/pathohahn/arn-frag.htm (11 de noviembre de 2013).

Sugier, D., P. Sugier and U. Gawlik-Dziki. 2013. Propagation and Introduction of Arnica montana L. into Cultivation: A Step to Reduce the Pressure on Endangered and High-Valued Medicinal Plant Species. The Scientific World Journal. DOI. 414363.
The Plants List. 2013. A working list of all plant species. Version 1. 1. http://www. theplantlist.org/ (21 de enero de 2014)

Tropicos. 2014. Data Base. Name Search. www.tropicos.org/Name/2701747 (21 de enero de 2014)

United States Department of Agriculture- Agricultural Research Service (USDAARS). 2013. National Genetic Resources Program. Germplasm Resources Information Network - [GRIN] [On Line Data Base]. 2013. National Germplasm Resources Laboratory.. http://www.ars-grin. gov/cgi-bin/npgs/html/tax _ search.pl (1 de noviembre de 2013).

Vogel, A. 2013. Arnica montana L. In: Plant encyclopedia. http://www.avogel. com/plant-encyclopaedia/arnica _ montana.php (27 de enero de 2014)

Waizel, B. J. 1995. Algunas notas sobre la planta medicinal Arnica montana L. Revista Médica del Instituto Mexicano del Seguro Social. 33 (3): $306,312,326$.

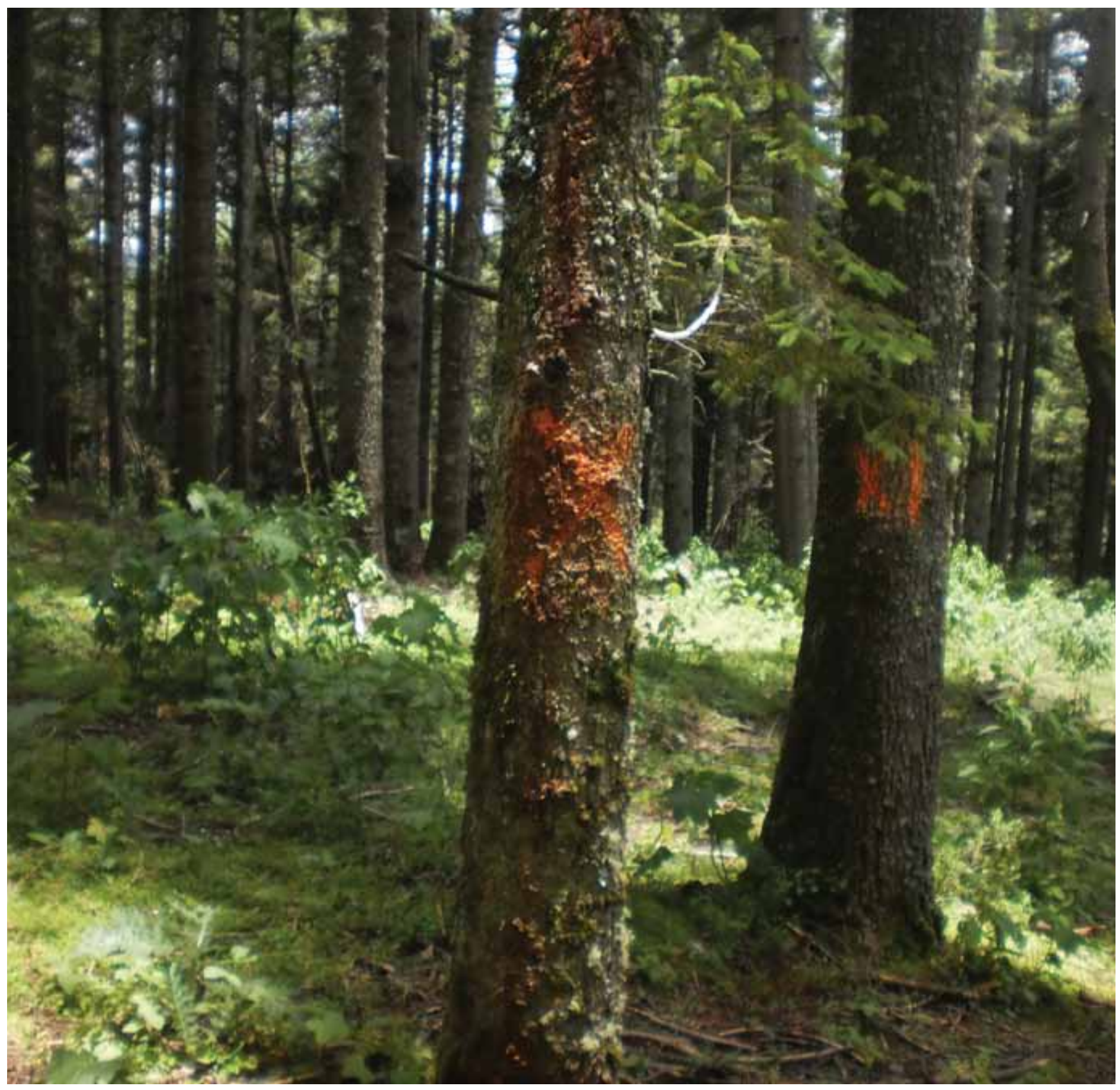

